\title{
Confabulations related to tacit awareness in visual neglect
}

\author{
L. Manning and L.D. Kartsounis
}

National Hospital for Neurology and Neurosurgery, Queen Square, London WC1 3BG, UK

Correspondence to: L. Manning at above address

\begin{abstract}
The case of a man with left-sided visuospatial neglect is reported. His performance on a test for neglect, the "burning house test", is analysed and compared with that of other patients referred to in the literature. We suggest that the confabulations neglect patients may produce when confronted with this test reflect the degree of preconscious awareness for left-sided features.
\end{abstract}

Keywords: Confabulations - Tacit awareness - Visual neglect

\section{INTRODUCTION}

Different kinds of responses have been reported in patients with visual neglect when they are confronted with pairs of line drawings which are identical in all respects, apart from one (crucial) feature placed on the "neglected" side. A specific type of test which is known to elicit so-called "tacit awareness" (i.e. an implied processing of a leftsided feature where conscious identification is absent) was described for the first time by Marshall and Halligan (1988). Their patient was unable to identify any difference between two houses whose crucial differing feature was the presence of bright red flames on the left side of one of the houses (Fig. 1). Yet, when asked to state in which house she would prefer to live, she consistently selected the non-burning house. Bisiach and Rusconi (1990) presented the same pair of houses (amongst other stimuli) to four patients. They obtained similar results to those by Marshall and Halligan (1988) in the first part of the test, i.e. their patients failed to detect the relevant difference between the two stimuli. Some response variations were, however, produced at this stage; that is, insignificant and/or non-existent differences were reported in the nonneglected, right side of the line drawings. In the second part of the test, in which the patient was asked to select the house in which he/she would prefer to live, different patterns of response were elicited; thus, one patient refused to answer the question ("since they were identical houses"), a second patient gave inconsistent answers and the remaining two patients consistently expressed preference for the burning house.

We describe a patient whose performance on the "burning house test" suggests that the confabulations neglect patients make in the second part of the test appear to reflect different degrees of perceptual awareness/unawareness concerning the neglected portion of the stimulus.

\section{CASE REPORT}

A.L., a 64 year old male patient, was admitted to the National Hospital, Queen Square, on 28 December 1992. He underwent a craniotomy on 23 February 1993 for evacuation of right-sided subdural empyema which extended over the frontal, temporal, parietal and occipital areas, its thickest depth being within the parietal region. There was a considerable degree of midline shift to the left with compression of the right lateral ventricle. Postoperatively he continued to present left homonymous hemianopsia. On neuropsychological assessment the patient did not present any signs of language impairment. His recognition memory for both verbal and visual material was impaired. Performance on visuoperceptual and visuospatial tests was poor but this appeared to be confounded by his visual neglect. On certain tests of frontal lobe dysfunction results were uneven, suggestive of anterior cerebral involvement.

A.L. presented a marked degree of visuospatial neglect on the left. On line bisection tasks he bisected all the lines significantly to the right side. On the Albert test of line cancellation he crossed out 18 of 25 lines on the right side of the midline and 0 of 25 on the left half. Similarly, on a cancellation task consisting of simple geometrical shapes, he failed to cross out any of the 15 circles on the left side of the midline while on the right side he cancelled 11 out of 15 . When asked to copy a drawing of a potted plant with two main branches (Marshall and 

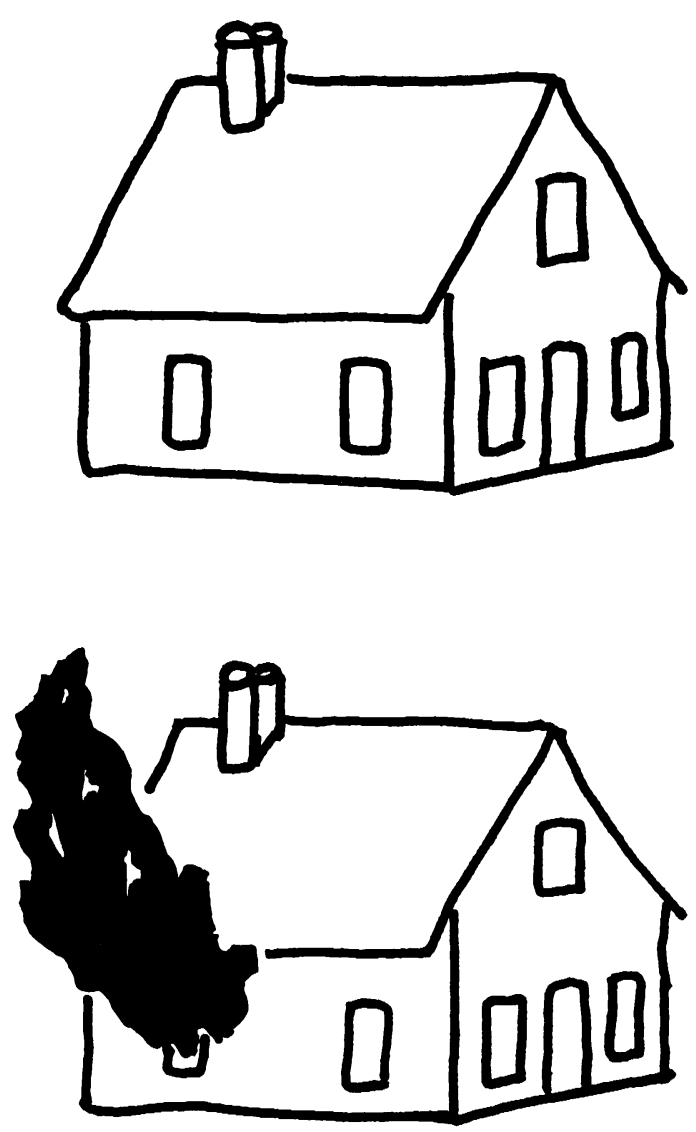

FIG. 1. The "burning house test" (adapted from Marshall and Halligan, 1988 and Bisiach and Rusconi, 1990).

Halligan, 1993), he omitted the upper part of the left branch. On two visual search tasks (involving either objects or numbers) he needed substantially more time to locate targets placed on the left than the right side of the page (mean time for locating objects: right side $1.2 \mathrm{~s}$; left $24 \mathrm{~s}$; for numbers: right side $2.5 \mathrm{~s}$; left $16 \mathrm{~s}$-total number of trials 22).

\section{Burning house test}

The pictures of the two houses were presented three times and the following observations were made:

\section{Test 1 (16 March 1993)}

Q: Are the houses the same?

R: Yes, they are the same.

Q: Are they exactly the same?

R: Yes.

Q: Where would you like to live?

$\mathrm{R}$ : [Points to the non-burning house]

Q: Why?

R: There is an extra fireplace, it's the heating, it's warmer and I wouldn't have to buy a wood-burning stove.
Test 2 (17 March 1993)

Q: Are the houses the same?

R: No, they are not.

Q: What is the difference?

$\mathrm{R}$ : There are other, extra fireplaces [pointing to the nonburning house] and two chimneys ... the major difference.

Q: Where would you like to live?

$\mathrm{R}$ : Live in this one [non-burning house].

Q: Why?

$\mathrm{R}$ : Because I can heat cheaper.

Test 3 (17 March 1993)

Q: Are the houses the same?

R: No, I don't think so.

$\mathrm{Q}:$ What is the difference?

$\mathrm{R}$ : It could be the amount, the number of windows ...

Q: Any other difference?

$\mathrm{R}$ : One is on fire and the other is not.

Q: Where would you like to live?

$\mathrm{R}$ : [Points to the non-burning house]

Q: Why?

R: Because it's not burning.

\section{COMMENT}

On a variety of tasks, including line bisection, cancellation, visual search and drawing tests, A.L.'s performance indicated a rather marked degree of left-sided visuospatial neglect. However, it is his performance on the "burning house test" which is of particular interest and merits special consideration. As in previous case reports, our patient's responses during the first two test trials implied "tacit awareness" of the house in flames in that he referred to "fireplaces" and "heating" arrangements, albeit within the non-burning house. This apparent transposition of the presence of flames from one house to the other may be seen as an analogous, although more intriguing, phenomenon to the transposition of features of the left side to the right side by neglect patients when copying complex visual stimuli (Marshall and Halligan, 1993).

In comparing the responses of previously reported patients with those of A.L., a graded pattern of performance emerges. P.G., Marshall and Halligan's (1988) patient, hardly justified her choice of a house to live in; furthermore, she did not become fully aware of the flames until the 21 st presentation of the stimuli and only after she had been primed with stimuli presenting one of the houses with flames on the right side. By contrast, R.C., Bisiach and Rusconi's (1990) patient, referred to non-existent characteristics, i.e. he confabulated when asked to explain his choice, in the second part of the test. For instance, he referred to the roof as being "especially convenient for rainy days", amongst other confabulatory responses. Nevertheless, R.C. appeared to be very close to awareness 
since he conjectured "maybe there is something here, but what is it?" Finally, A.L., our patient, who did not acknowledge any difference between the houses initially, did make comments relating to the presence of fire during the second part of the test, and became fully aware of it after only three presentations of the stimuli.

An analysis of the pattern of responses of these three aforementioned patients provides additional support and extends the notion of Bisiach and Rusconi (1990) suggesting that the presence of confabulations in some of their patients could be induced by left-sided features of stimuli of which these patients seem to be unaware. Covert knowledge is probably present in most visual neglect patients tested with the "burning house test". However, the likelihood of preconscious processes to become conscious and the number of exposures to the stimuli a patient may need in order to detect the left-sided flames vary amongst different patients. In the light of the previous case studies and the present report, we would like to suggest that a higher or more extensive preconscious access to output (Marshall and Halligan, 1988) is indicated by the production of confabulations related to the crucial feature on the left side in one of the stimuli.

\section{Acknowledgement}

We wish to thank Professor E.K. Warrington for useful comments on the manuscript of this paper.

\section{REFERENCES}

Bisiach E and Rusconi ML (1990) Break-down of perceptual awareness in unilateral neglect. Cortex, 26, 643-649.

Marshall JC and Halligan P (1988) Blindsight and insight in visuo-spatial neglect. Nature, 336 (22/29), 766-767.

Marshall JC and Halligan P (1993) Visuo-spatial neglect: A new copying test to assess perceptual parsing. Journal of Neurology, 40, 37-40.

(Received 1 July 1993; accepted 12 August 1993) 


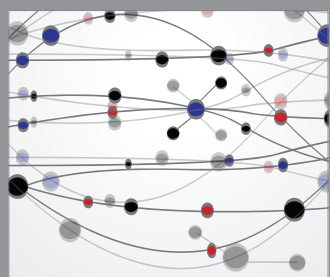

The Scientific World Journal
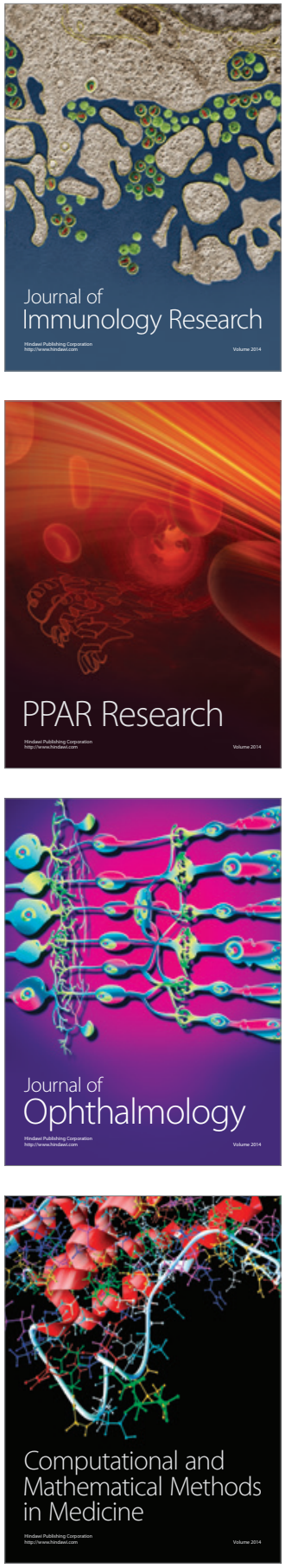

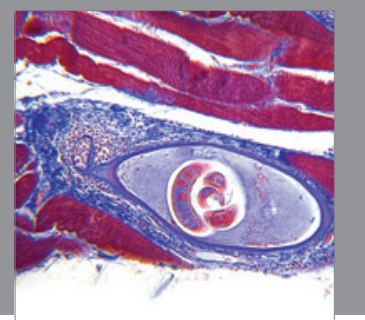

Gastroenterology

Research and Practice
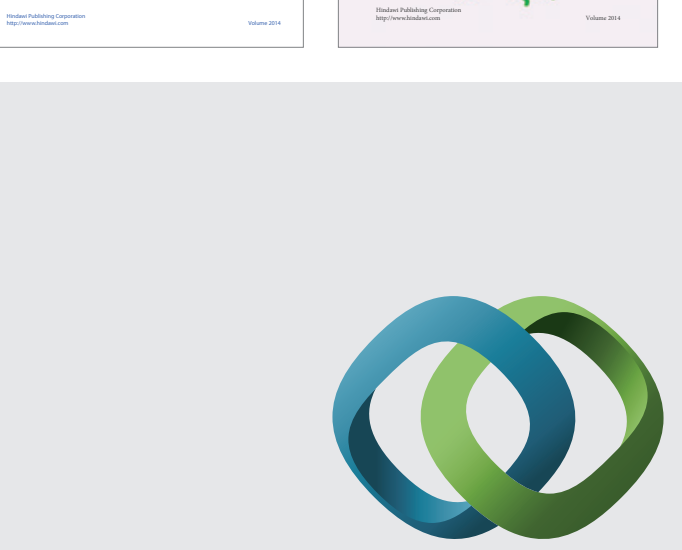

\section{Hindawi}

Submit your manuscripts at

http://www.hindawi.com
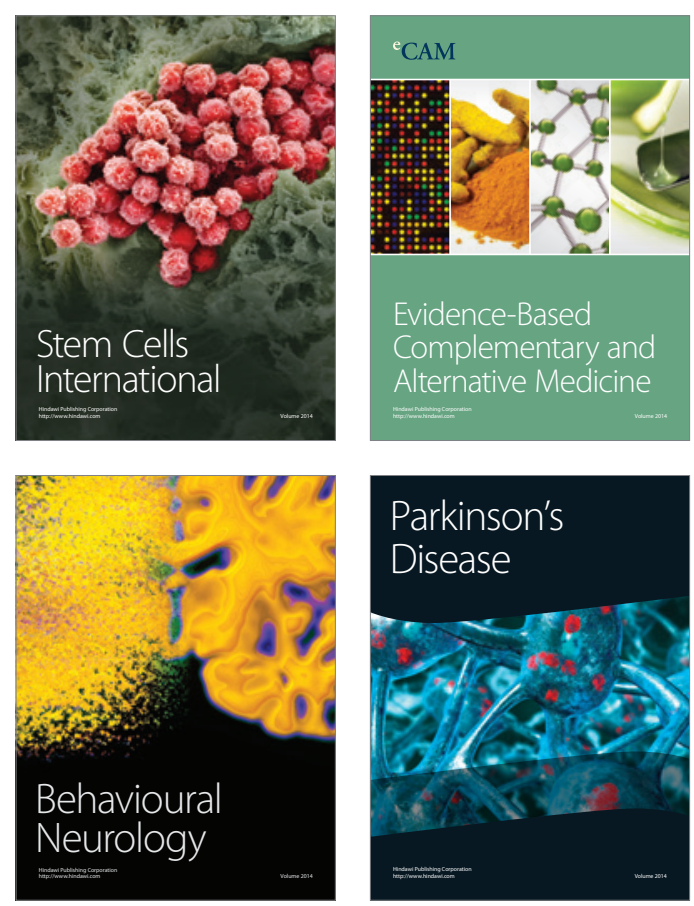

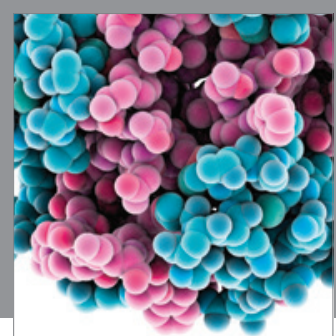

Journal of
Diabetes Research

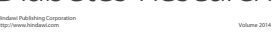

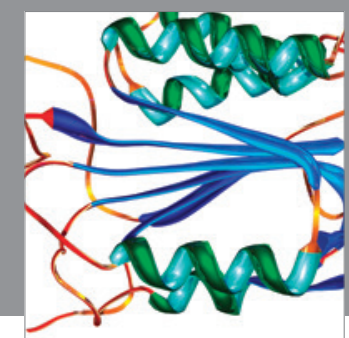

Disease Markers
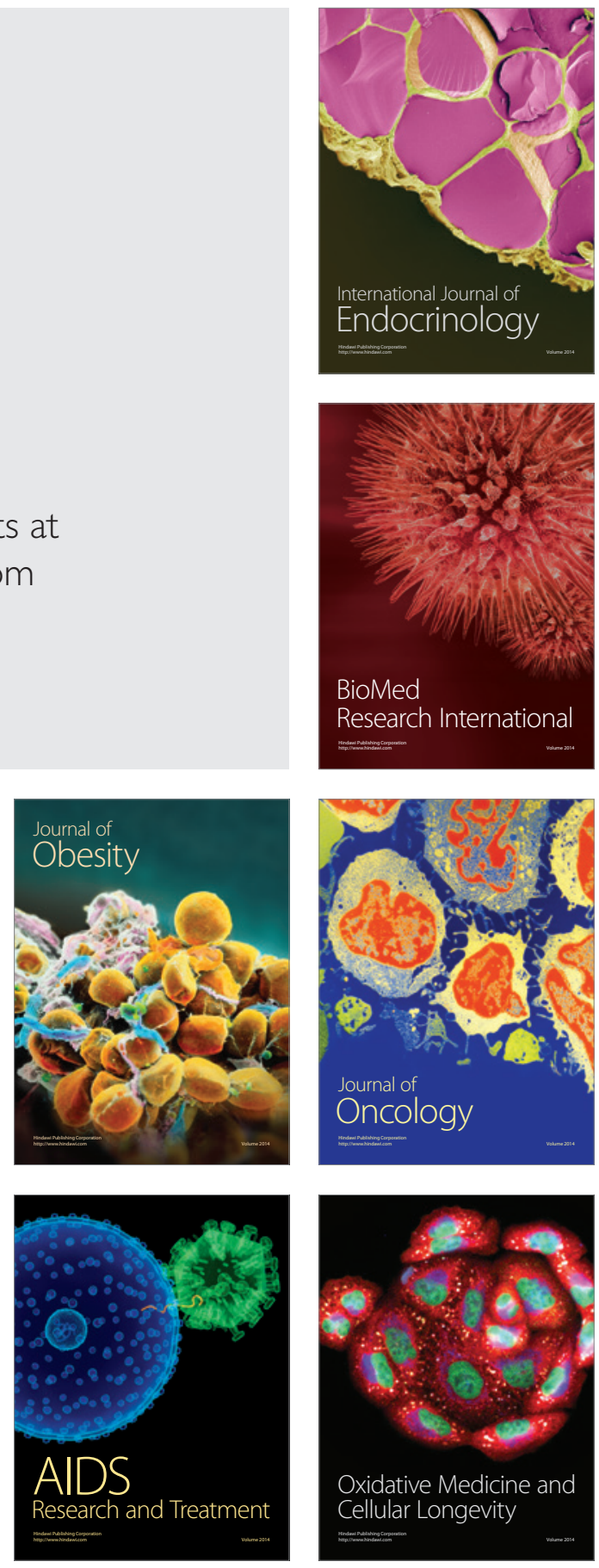